Article

\title{
Liver Transplantation Is Highly Effective in Children with Irresectable Hepatoblastoma
}

\author{
Simon Moosburner ${ }^{1,2, * \mathbb{D}}$, Moritz Schmelzle ${ }^{1}$, Wenzel Schöning ${ }^{1}$, Anika Kästner ${ }^{1}$, Philippa Seika ${ }^{1}$,

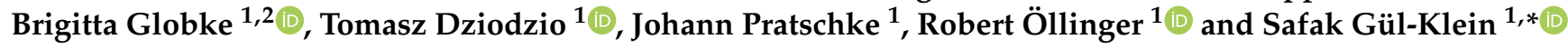 \\ 1 Deparment of Surgery, Campus Charité Mitte and Campus Virchow-Klinikum, \\ Charité-Universitätsmedizin Berlin, 13353 Berlin, Germany; moritz.schmelzle@charite.de (M.S.); \\ wenzel.schoening@charite.de (W.S.); anika.kaestner@med.uni-greifswald.de (A.K.); \\ philippa.seika@charite.de (P.S.); brigitta.globke@charite.de (B.G.); tomasz.dziodzio@charite.de (T.D.); \\ johann.pratschke@charite.de (J.P.); robert.oellinger@charite.de (R.Ö.) \\ 2 BIH Charité (Digital) Clinician Scientist Program, Berlin Institute of Health, 10178 Berlin, Germany \\ * Correspondence: simon.moosburner@charite.de (S.M.); safak.guel@charite.de (S.G.-K.); \\ Tel.: +01-76-3452-1755 (S.M.); +49-17-0740-2409 (S.G.-K.)
}

check for updates

Citation: Moosburner, S.; Schmelzle, M.; Schöning, W.; Kästner, A.; Seika,

P.; Globke, B.; Dziodzio, T.; Pratschke,

J.; Öllinger, R.; Gül-Klein, S. Liver

Transplantation Is Highly Effective in Children with Irresectable

Hepatoblastoma. Medicina 2021, 57, 819. https://doi.org/10.3390/

medicina57080819

Academic Editor: Giovanni Tarantino

Received: 29 July 2021

Accepted: 8 August 2021

Published: 12 August 2021

Publisher's Note: MDPI stays neutral with regard to jurisdictional claims in published maps and institutional affiliations.

Copyright: (c) 2021 by the authors. Licensee MDPI, Basel, Switzerland. This article is an open access article distributed under the terms and conditions of the Creative Commons Attribution (CC BY) license (https:// creativecommons.org/licenses/by/ $4.0 /)$.

\begin{abstract}
Background and Objectives: In children, hepatoblastoma preferentially is managed by liver resection (LR). However, in irresectable cases, liver transplantation (LT) is required. The aim of our study was to compare short- and long-term results after LR and LT for the curative treatment of hepatoblastoma. Materials and Methods: Retrospective analysis of all patients treated surgically for hepatoblastoma from January 2000 until December 2019 was performed. Demographic and clinical data were collected before and after surgery. The primary endpoints were disease free survival and patient survival. Results: In total, 38 patients were included into our analysis $(n=$ 28 for LR, $n=10$ for LT) with a median follow-up of 5 years. 36 patients received chemotherapy prior to surgery. Total hospital stay and intensive care unit (ICU) stay were significantly longer within the LT vs. the LR group (ICU 23 vs. 4 days, hospital stay 34 vs. 16 days, respectively; $p<0.001$ ). Surgical complications ( $\leq$ Clavien-Dindo 3a) were equally distributed in both groups ( $60 \%$ vs. $57 \%$; $p=1.00$ ). Severe complications ( $\geq$ Clavien-Dindo 3a) were more frequent after LT ( $50 \%$ vs. $21.4 \%$; $p=0.11$ ). Recurrence rates were $10.7 \%$ for LR and $0 \%$ for LT at 5 years after resection or transplantation $(p=0.94)$. Overall, 5 -year survival was $90 \%$ for LT and $96 \%$ for LR $(p=0.44)$. Conclusions: In irresectable cases, liver transplantation reveals excellent outcomes in children with hepatoblastoma with an acceptable number of perioperative complications.
\end{abstract}

Keywords: hepatoblastoma; pediatric liver transplantation; pediatric liver resection; survival; postoperative complications

\section{Introduction}

Primary hepatic tumors in children account for about $1 \%$ of all childhood malignancies. Hepatoblastoma is the most common malignant hepatic tumor, accounting for $90 \%$ of all malignant hepatic tumors in children [1,2]. Despite hepatoblastoma remaining a relatively rare disease, hepatoblastoma has shown an annual increase in incidence of $4.3 \%$ in children under the age of 19, especially in countries with a high human development index [3-5].

Patients generally remain asymptomatic and are diagnosed in a progressed state of the disease, requiring a multimodal treatment strategy of chemotherapy (CTx) and surgery. Staging is performed through the PRE-Treatment EXTent of disease (PRETEXT) system, a radiological score for risk stratification prior to neoadjuvant chemotherapy influencing the method of surgical treatment [6].

Over the past three decades, several multicenter study groups have introduced effective risk-based chemotherapeutic regimens, which in combination with more aggressive 
surgical approaches, including liver transplantation (LT), have resulted in significant improvements in outcomes [7-12]. Nevertheless, Hiyama et al., for the Japanese Study Group for Pediatric Liver Tumors, recently showed that neoadjuvant and adjuvant CTx with cisplatin-tetrahydropyranyl-adriamycin (pirarubicin; CITA) was effective for resectable hepatoblastoma, but remained unsatisfactory for irresectable and metastatic hepatoblastoma patients [12]. After CTx, if hepatoblastoma remains, surgical completion through liver resection (LR) or LT in the following phase can lead to a completely curative treatment [13].

Primary resectability implies radical surgical resection as a first-line therapy. Nevertheless, curative LR after successful tumor reduction remains a challenge, especially in cases of extensive liver resection. LT is in turn the treatment of choice for irresectable tumors (bilobar localization, vascular infiltration) and in cases of expected excessive parenchymal loss by radical resection [14-17]. Overall, an improvement in survival rates, with 5-year survival rates as high as $90 \%$, has been observed over the past four decades $[18,19]$. Advanced hepatoblastoma requires a decision to be made between a complex and radical LR or alternatively a LT, which is also known as a complex procedure-especially in infants. LT as well as extended resections for metastatic and locally advanced hepatoblastoma after neoadjuvant chemotherapy require referral to centers with expertise in both pediatric transplantation and hepatobiliary surgery. Preoperative imaging may identify hepatoblastomas as presumptively inoperable and subvert surgical exploration. Synchronous pulmonary metastases are seen in approximately $20 \%$ of cases and are not, per se, a contraindication for a radical surgical approach; however, this is an ongoing matter of discussion [20].

The aim of our study was to evaluate patients after LR and after LT in the postoperative course with respect to early and late complications and recurrence of hepatoblastoma.

\section{Materials and Methods}

\subsection{Study Design and Participants}

All patients with hepatoblastoma presenting at the Surgical Department, Campus Charité Mitte and Campus Virchow-Klinikum at Charité-Universitätsmedizin Berlin from 1990 to 2019 were included in the study.

Demographic and clinical data before and after surgery, i.e., age at presentation, gender, tumor size, presence of distant metastasis, values of alpha-fetoprotein (AFP), chemotherapy, type of surgery, postoperative complications, complication management, tumor recurrence/disease free survival, and mortality for both groups were collected. Patients were followed up at regular time intervals through routine follow-up examinations. In addition, all unplanned inpatient stays were considered for both groups, with special attention given to readmissions that entailed reoperations or were associated with complications in general. PRETEXT staging was performed by our radiologists at the time of diagnosis according to standardized criteria [6]. Postoperative complications were classified after Clavien-Dindo. Cancer recurrence was defined as local recurrence or distant metastasis.

Immunosuppression was administered based on individualized protocols including calcineurin inhibitors, mycophenolate mofetil (MMF), mechanistic targets of rapamycin (mTOR) inhibitors and steroids.

Patients who underwent LR before LT were included in the LT group. The study was approved by the institutional ethics board (EA2/267/20).

\subsection{Surgical Technique}

LTs from deceased donors were donations after brain death only (DBD) due to regulations in Germany. DBD donors were chosen based on donor age, size of the donor organ, cause of death, laboratory values at time of organ donation, perspective cold ischemia time and frozen cut section analysis, if available. Living donors for partial living donor liver transplantation (LDLT) were carefully evaluated, including by the ethics board of the German federal medical association ("Bundesärztekammer"), and underwent S2/3 sectionectomy using a small median laparotomy. Full-size organs were transplanted or- 
thotopically, whereas split grafts and grafts form living donors were placed in a modified piggy-back technique, using the recipient's unified left and middle hepatic vein for venous anastomosis. Portal vein and arterial anastomoses were all performed in an end-to-end fashion using loupes or a microscope for magnification. Biliary anastomosis was performed in a side-to-side technique with the addition of a T-Drain. If duct-to-duct anastomosis was not feasible, a hepaticojejunostomy was performed with intraoperative placement of a Polyvinyl drainage.

LR was performed as open surgery with a right subcostal incision and upper midline extension to the xiphoid. Intraoperative ultrasound was used to identify a dissection plane. Pringle's maneuver was prepared for use if needed. Parenchymal dissection was carried out using ultrasound dissection.

\subsection{Statistical Analysis}

Statistical analysis was performed using the software solutions $R$ (version 4.0.3) and R Studio (version 1.25) for macOS (both: R Foundation for Statistical computing, Vienna, Austria). Graphs were plotted using GraphPad PRISM version 8.2 for macOS (GraphPad Software, La Jolla, CA, USA). Survival was analyzed using the Kaplan-Meier curves and compared with the log rank method. Data were tested for normality using the ShapiroWilk test and analyzed with a student's t-test or Mann-Whitney U test accordingly. Data, unless otherwise stated, are reported as mean and standard deviation (SD) or median and interquartile range (IQR).

\section{Results}

\subsection{Patient Characteristics}

During the analysis period, 42 patients with suspected hepatoblastoma were surgically treated in the Charité-Universitätsmedizin Berlin. Of those 42 patients, 4 were excluded from our analysis due to final histopathology showing no hepatoblastoma $(n=2)$ or patients being lost to follow-up $(n=2)$. Median age at time of presentation was 2 years (IQR 1 year). Median follow-up was 64 months for LR and 170 months for LT patients respectively (Table 1).

Table 1. Liver Transplantation and Liver Resection compared.

\begin{tabular}{|c|c|c|c|}
\hline & Liver Resection & Liver Transplantation & \multirow{2}{*}{$p$} \\
\hline & $N=28$ & $N=10$ & \\
\hline Sex $[f]$ & $10(35.7 \%)$ & $7(70.0 \%)$ & 0.078 \\
\hline Age [years] & $1.00[0.00 ; 2.00]$ & $2.00[2.00 ; 3.00]$ & 0.069 \\
\hline Age Groups [years] & & & 0.328 \\
\hline$<1$ & $9(32.1 \%)$ & $1(10.0 \%)$ & \\
\hline $1-2$ & $13(46.4 \%)$ & $5(50.0 \%)$ & \\
\hline$\geq 3$ & $6(21.4 \%)$ & $4(40.0 \%)$ & \\
\hline PrēẼEX & & & 0.002 \\
\hline 1 & $7(25.9 \%)$ & $0(0.00 \%)$ & \\
\hline 2 & $10(37.0 \%)$ & $0(0.00 \%)$ & \\
\hline 3 & $9(33.3 \%)$ & $5(55.6 \%)$ & \\
\hline 4 & $1(3.70 \%)$ & $4(44.4 \%)$ & \\
\hline Metastasis & $8(28.6 \%)$ & $3(30.0 \%)$ & 1.000 \\
\hline Metastasis Localization & & & 0.101 \\
\hline bone and spinal & $1(3.57 \%)$ & $0(0.00 \%)$ & \\
\hline lung & $7(25.0 \%)$ & $1(10.0 \%)$ & \\
\hline Operative Time [min] & $168(50.5)$ & $392(103)$ & $<0.001$ \\
\hline Hospital Stay [d] & $15.5[10.0 ; 21.2]$ & $34.0[28.0 ; 44.0]$ & 0.001 \\
\hline Intensive Care Unit [d] & $3.50[2.00 ; 6.00]$ & $23.0[19.0 ; 29.0]$ & $<0.001$ \\
\hline Complications & $16(57.1 \%)$ & $6(60.0 \%)$ & 1.000 \\
\hline
\end{tabular}


Table 1. Cont.

\begin{tabular}{|c|c|c|c|}
\hline & Liver Resection & Liver Transplantation & \multirow{2}{*}{$p$} \\
\hline & $N=28$ & $N=10$ & \\
\hline Clavien-Dindo & & & 0.087 \\
\hline 0 & $12(42.9 \%)$ & $4(40.0 \%)$ & \\
\hline 1 & $2(7.14 \%)$ & $0(0.00 \%)$ & \\
\hline 2 & $8(28.6 \%)$ & $1(10.0 \%)$ & \\
\hline $3 a$ & $1(3.57 \%)$ & $0(0.00 \%)$ & \\
\hline $3 b$ & $2(7.14 \%)$ & $5(50.0 \%)$ & \\
\hline $4 a$ & $3(10.7 \%)$ & $0(0.00 \%)$ & \\
\hline Follow-up in years & $5.00[3.00 ; 7.00]$ & $14.0[4.25 ; 18.5]$ & 0.093 \\
\hline Neoadjuvant Chemotherapy & $27(96.4 \%)$ & $9(90 \%)$ & 0.43 \\
\hline Adjuvant Chemotherapy & $27(96.4 \%)$ & $1(12.5 \%)$ & $<0.001$ \\
\hline AFP & & & 0.178 \\
\hline$<999$ & $0(0.00 \%)$ & $1(12.5 \%)$ & \\
\hline 1000-9999 & $5(20.8 \%)$ & $0(0.00 \%)$ & \\
\hline $10,000-99,999$ & $6(25.0 \%)$ & $4(50.0 \%)$ & \\
\hline $100,000-999,999$ & $11(45.8 \%)$ & $3(37.5 \%)$ & \\
\hline$>1,000,000$ & $2(8.33 \%)$ & $0(0.00 \%)$ & \\
\hline
\end{tabular}

AFP: alpha-fetoprotein.

Ten patients (26\%) underwent LT for hepatoblastoma. Within these 10 patients, 5 patients received a graft from a living donor, the remaining 5 patients received either a segment II/III split graft $(n=3)$ or a full-size graft $(n=2)$ from a deceased donor. For three patients, LT was a rescue procedure due to recurrent disease, small for size syndrome, or cirrhosis in the remnant liver. Immunosuppression in the 10 LT patients was carried out as monotherapy with tacrolimus (tac) in 5 patients, while 3 patients had dual-therapy (Tacrolimus and plus Mycophenolate mofetil). One patient received monotherapy with Everolimus. A total of 28 patients underwent LR, amongst them 1 patient with a segment II resection, 3 patients with atypical resections including $>2$ segments, 4 patients with left-lateral hepatectomy (SII and III), 4 patients with left hemihepatectomy, 7 patients with right hemihepatectomy, 5 patients with extended right hemihepatectomy and 4 patients with extended left hemihepatectomy.

\subsection{Tumor Characteristics}

Histopathological analysis showed most tumors being of fetal origin ( $54 \%$ in LR group, $50 \%$ ins LT group) and roughly a third of mixed origin. Macroscopical tumor-free resection margins were achieved in all LT and LR patients. In four patients (14.3\%) after LR, there was microscopical invasion by tumor cells in the resection margins (R1). Synchronous distant metastases did not differ between groups and affected one third of patients $(p=1.00)$. There were mostly lung metastases $(n=7)$ in the LR group, and one bone and one spinal cord metastasis. One patient in the LT group had pulmonary metastases, and two patients lymphonodal metastases. Almost all $(n=27,96.4 \%)$ patients after LR received adjuvant chemotherapy vs. $10 \%$ in the patient group after liver transplantation.

\subsection{Perioperative Complications}

Surgical complications ( $\leq$ Clavien-Dindo 3a) were equally distributed in both groups ( $60 \%$ vs. $57 \% ; p=1.00)$. Severe complications $\geq$ Clavien-Dindo 3 a were more frequent in patients after LT (Table 1$)$. Complications after LT were biliary complications $(n=3$, $30.0 \%)$, hemorrhage $(n=2,20 \%)$, and portal vein thrombosis $(n=2 ; 20 \%)$. After LR, biliary complications were most common $(n=5 ; 17.9 \%$, vs. $30 \%$ for LT; $p=0.41)$, followed by hemorrhage $(n=3 ; 10.7 \%$, vs. $20 \%$ for LT; $p=0.59)$ (Table 2$)$. 
Table 2. Complications after Liver Transplantation and Liver Resection.

\begin{tabular}{ccc}
\hline & Liver Resection & Liver Transplantation \\
\cline { 2 - 3 } & $N=\mathbf{2 8}$ & $\mathbf{N}=\mathbf{1 0}$ \\
\hline Early Complications & & \\
Biliary & $5(17.86 \%)$ & $3(30 \%)$ \\
Vascular & $0(0 \%)$ & $2(20 \%)$ \\
Bleeding & $3(10.71 \%)$ & $2(20 \%)$ \\
Infection & $0(0 \%)$ & $1(10 \%)$ \\
Acute rejection & $0(0 \%)$ & $1(10 \%)$ \\
Other & $2(7.14 \%)$ & $0(0 \%)$ \\
Late Complications & & $0(0 \%)$ \\
Ototoxicity & $3(10.71 \%)$ & $1(10 \%)$ \\
Adhesions & $0(0 \%)$ & $1(10 \%)$ \\
Bile strictures & $0(0 \%)$ & $0(0 \%)$ \\
Recurrence & $2(7.14)$ & $0(0 \%)$ \\
\hline
\end{tabular}

One patient after LT had impaired renal function postoperatively without the necessity of dialysis. One patient suffered from rejected once resolved by corticosteroid pulse treatment. Another patient suffered from two phases of acute rejection, both successfully treated with steroids. One patient suffered from one instance of short-term leukopenia under tacrolimus therapy.

\subsection{Long Term Complications and Survival}

Two patients after LR suffered from chronic bile strictures and required frequent endoscopic therapy. Three patients in the LR group had tumor recurrence, one month, 6 months, and 8 months after resection, by the means of metachronous pulmonary metastases and, in one case, additional intrahepatic metastases.

The 1-, 5-, and 10-year recurrence-free rates were $89 \%, 89 \%$, and $89 \%$ for LR and $100 \%$, $100 \%$, and $100 \%$ for LT $(p=0.94)$. Overall, the $1-, 5-$, and 10 -year survival was $90 \%, 90 \%$, and $90 \%$ for LT and $96 \%, 96 \%$, and $96 \%$ for LR ( $p=0.44$ ) (Figure 1). One patient died after emergency adhesiolysis surgery as a result of his abdominal sepsis three months after LT. The second patient died due to septic shock caused to pneumococcal infection 23 years after LT.

A

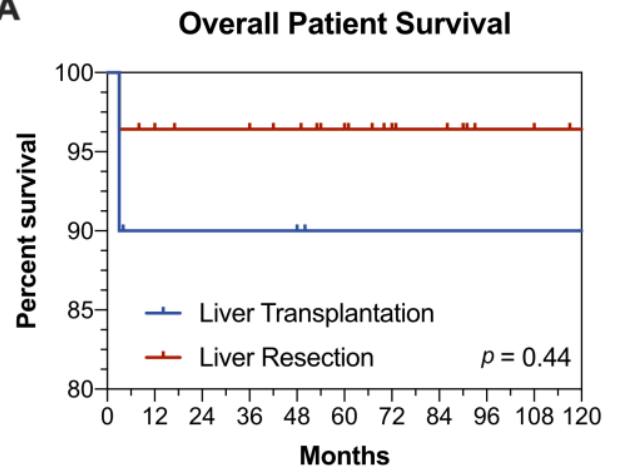

B

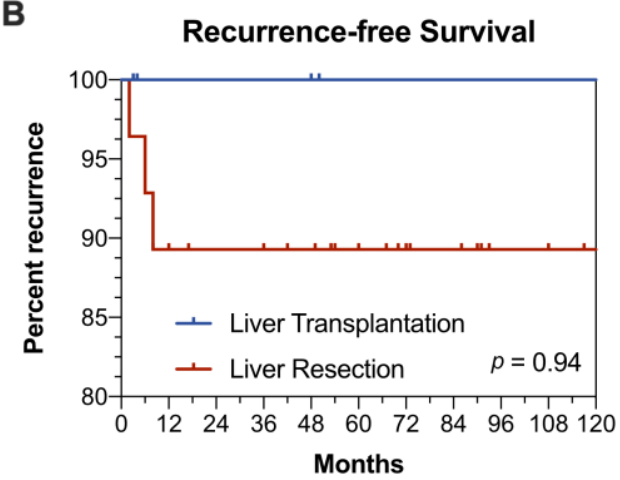

Figure 1. (A) Overall patient survival after liver resection or liver transplantation (B) Recurrence-free Survival.

One patient in the LR group, who had already undergone surgery in an M1 setting (pulmonary, osseous metastases), died 3 months after LR due to extensive intrahepatic recurrence after initial $\mathrm{R} 0$ resection, with all other patients being alive at the date of the last follow-up. 


\section{Discussion}

Hepatoblastoma is a rare disease, but it is the most frequent intrahepatic malignancy of the childhood. In general, hepatoblastomas are diagnosed at a late stage with often large size and frequent vascular infiltration [21]. Neoadjuvant CTx is the gold standard before surgical resection. Excellent results can be achieved with a monotherapy of cisplatin in standard risk tumors, cisplatin alternating with carboplatin-doxorubicin in high-risk tumors, and dose-intensive weekly cisplatin/doxorubicin induction therapy for very high risk tumors by the means of shrinkage of the primary tumor and control of distant metastasis [10]. A combination of chemotherapy and resection reaches survival rates of $81.5 \%$ and $81.0 \%$ at $5 / 10$ years [22,23]. However, despite advances in CTx, in certain cases, resection is not always feasible for anatomical or functional reasons, leaving LT as the only curative option [24]. We herein analyzed our own data comparing outcomes in children with hepatoblastoma after resection with LT.

Demographic data showed an equal distribution in both groups. As expected, in the LT cohort, disease severity according to PRETEXT was significantly higher. Similarly, Kulkarni et al. demonstrated that patients with bilobar involvement were more likely to receive LT based on an analysis of the National Cancer Database for surgical therapy of pediatric hepatoblastoma. However, patients in the LT group had a longer overall time from diagnosis to surgery associated with waiting list time in addition to neoadjuvant chemotherapy [25]. With respect to early and late complications, long-term course, and metastases, the results of our analysis showed an equal distribution in both groups. Interestingly, none of the patients developed hepatic local recurrence or intrahepatic metastasis after LT, and only three patients after LR.

Nevertheless, the decision of when to opt for surgical resection, LT, or CTx without surgical intervention can become a balancing act: In general, the basic recommendation for LT in PRETEXT stage IV tumors and centrally placed PRETEXT stage III tumors with infiltration of vascular structures after neoadjuvant CTx is primary resection [26]. Additionally, according to the current surgical guidelines of the Children's Oncology Group, children with suspected inoperable findings should be referred to a specialized center at an early stage, so that a decision between liver tumor resection and transplantation by a liver specialist can be made [27]. This is especially important considering that LT has to be considered as an emergency therapy after primary LR with a small for size liver remnant or in case of a relapse after LR, as described for one patient in our cohort. The decisionmaking process for the most suitable therapy after neoadjuvant CTx is determined by specific tumor characteristics, such as particularly hepatoblastoma size, critical anatomical localization for LR (involvement of vascular/bilary structures, multicenter localization), and probable function of the future liver remnant [17,28-30]. As Meyers et al. showed, the Children's Hepatic Tumors International Collaboration (CHIC) has created a unified global approach to risk stratification for children with hepatoblastoma [31]. Regardless of this, molecular biological studies on prognostic tumor markers may play a decisive role in risk stratification and optimized patient selection in the future and will have the potential to increase the number of primarily resectable patients.

Excellent tumor control was achieved in our series with the combination of CTx and LT, considering that immunosuppression would be assumed, bearing a higher recurrence risk (e.g., hepatocellular carcinoma in adults) [32]. There was no incidence of local recurrence or secondary malignant tumors in our LT group.

Due to concerns for the transplanted graft, only one patient received adjuvant chemotherapy after LT (i.e., our patient with pulmonary metastases). In contrast, $96.4 \%$ of the patients in the LR group received adjuvant treatment. This is of special interest, as the recurrence rate was $0 \%$ in the LT group. While there is no unique strategy or consensus on adjuvant chemotherapy after LT, admittedly there are centers following this concept. Without any decisive evidence, this decision probably has to be made on an individualized level. SIOPEL-1 and the World Experience Review showed superior survival rates of $85 \%$ at 10 years and $82 \%$ at 10 years after primary transplantation [22,23]. Zsiros et al. showed in 
the SIOPEL-3 and-4 studies that transplantation was associated with a 75\% 3-year survival rate $[33,34]$.

With respect to peri- and post-operative complications, these were comparable in our cohort between both groups. However, as expected, operative time, intensive care unit (ICU) stay and hospital stay were considerably longer in the LT group, matching the study by Kulkarni et al. [25]. In general, outcomes after LT in pediatric patients were superior to adults, independent of donor or indication for transplantation, reaching 1-year survival rates of up to $95 \%$ [35]. Adults undergoing LT for malignant tumors had unsatisfactory outcomes, in part due to tumor recurrence. Certainly, hepatocellular carcinoma or cholangiocellular carcinoma cannot be directly compared to hepatoblastoma with respect to long term recurrence outcomes; however, hepatoblastoma patients must be considered for LT in irresectable cases, despite donor organ scarcity due to the excellent results.

The limitations of our study are the small sample size, the retrospective study design, and the variance in the different therapy strategies. However, data to hepatoblastoma are not widely published, and we feel that, indeed, our study adds to previously published research.

\section{Conclusions}

In conclusion, LT for hepatoblastoma, if possible, provides excellent oncological results with no recurrence to report in our study cohort. Despite overall longer length of intensive care unit and hospital stay, severe complications ( $\geq$ Clavien-Dindo 3a) were only slightly more frequent for patients receiving LT. Overall, 5-year survival surpassed $90 \%$ for either treatment option. To choose the most suitable treatment option for each patient, we want to highlight the necessity of a detailed assessment by an experienced interdisciplinary team in a center for hepato-pancreatico-biliary surgery, with pediatric and transplant surgery, for optimal results in LT or LR in hepatoblastoma, especially in advanced cases.

Author Contributions: Investigation, S.G.-K.; writing—original draft preparation and review and editing, S.M., S.G.-K.; review and editing, R.Ö.; methodology, data curation, A.K., P.S.; supervision, B.G., T.D.; M.S., W.S., J.P. All authors have read and agreed to the published version of the manuscript.

Funding: This research was funded by BIH-Charité Clinician Scientist Program funded by the Charité-Universitätsmedizin Berlin and the Berlin Institute of Health and the APC was funded by Charite-Universitätsmedizin Berlin.

Institutional Review Board Statement: The study was conducted according to the guidelines of the Declaration of Helsinki, and approved by the Ethics Committee of Charité-Universitätsmedizin Berlin (EA2/267/20; 25 March 2021).

Informed Consent Statement: Patient consent was originally obtained as part of intimate hospitalization, so no repeat consent was obtained as part of the retrospective analysis. In addition, this is a historical cohort with very long follow-up. In this respect, the original consents for the analysis of the patient data are no longer available in some cases, as they were not transferred to the electronic files. In addition, some of the patients are also international patients, not all of whom live in Germany or are affiliated with our university hospital.

Data Availability Statement: The data presented in this study are available on request from the corresponding author. The data are not publicly available due to the informed consent obtained from all subjects.

Acknowledgments: In We acknowledge support from the German Research Foundation (DFG) and the Open Access Publication Fund of Charité- Universitätsmedizin Berlin for open access publication costs.

Conflicts of Interest: The authors declare no conflict of interest.

\section{References}

1. Litten, J.B.; Tomlinson, G.E. Liver Tumors in Children. Oncology 2008, 13, 812-820. [CrossRef]

2. Darbari, A. Epidemiology of primary hepatic malignancies in U.S. children. Hepatology 2003, 38, 560-566. [CrossRef]

3. Hadzic, N.; Finegold, M.J. Liver Neoplasia in Children. Clin. Liver Dis. 2011, 15, 443-462. [CrossRef] 
4. Hubbard, A.K.; Spector, L.G.; Fortuna, G.; Marcotte, E.L.; Poynter, J.N. Trends in International Incidence of Pediatric Cancers in Children Under 5 Years of Age: 1988-2012. JNCI Cancer Spectr. 2019, 3, pkz007. [CrossRef]

5. Allan, B.J.; Parikh, P.P.; Diaz, S.; Perez, E.A.; Neville, H.L.; Sola, J.E. Predictors of survival and incidence of hepatoblastoma in the paediatric population. HPB 2013, 15, 741-746. [CrossRef] [PubMed]

6. Towbin, A.J.; Meyers, R.L.; Woodley, H.; Miyazaki, O.; Weldon, C.B.; Morland, B.; Hiyama, E.; Czauderna, P.; Roebuck, D.J.; Tiao, G.M.; et al. 2017 PRETEXT: Radiologic staging system for primary hepatic malignancies of childhood revised for the Paediatric Hepatic International Tumour Trial (PHITT). Pediatr Radiol. 2018, 48, 536-554. [CrossRef] [PubMed]

7. Perilongo, G.; Shafford, E.; Plaschkes, J. SIOPEL trials using preoperative chemotherapy in hepatoblastoma. Lancet Oncol. 2000, 1, 94-100. [CrossRef]

8. Katzenstein, H.M.; London, W.B.; Douglass, E.C.; Reynolds, M.; Plaschkes, J.; Finegold, M.J.; Bowman, L.C. Treatment of Unresectable and Metastatic Hepatoblastoma: A Pediatric Oncology Group Phase II Study. JCO 2002, 20, 3438-3444. [CrossRef]

9. Sasaki, F.; Matsunaga, T.; Iwafuchi, M.; Hayashi, Y.; Ohkawa, H.; Ohira, M.; Okamatsu, T.; Sugito, T.; Tsuchida, Y.; Toyosaka, A.; et al. Outcome of hepatoblastoma treated with the JPLT-1 (Japanese Study Group for Pediatric Liver Tumor) Protocol-1: A report from the Japanese Study Group for Pediatric Liver Tumor. J. Pediatric Surg. 2002, 37, 851-856. [CrossRef] [PubMed]

10. Perilongo, G.; Maibach, R.; Shafford, E.; Brugieres, L.; Brock, P.; Morland, B.; de Camargo, B.; Zsiros, J.; Roebuck, D.; Zimmermann, A.; et al. Cisplatin versus Cisplatin plus Doxorubicin for Standard-Risk Hepatoblastoma. N. Engl. J. Med. 2009, 361, 1662-1670. [CrossRef] [PubMed]

11. Hishiki, T.; Matsunaga, T.; Sasaki, F.; Yano, M.; Ida, K.; Horie, H.; Kondo, S.; Watanabe, K.-I.; Oue, T.; Tajiri, T.; et al. Outcome of hepatoblastomas treated using the Japanese Study Group for Pediatric Liver Tumor (JPLT) protocol-2: Report from the JPLT. Pediatr Surg. Int. 2011, 27, 1-8. [CrossRef]

12. Hiyama, E.; Hishiki, T.; Watanabe, K.; Ida, K.; Ueda, Y.; Kurihara, S.; Yano, M.; Hoshino, K.; Yokoi, A.; Takama, Y.; et al. Outcome and Late Complications of Hepatoblastomas Treated Using the Japanese Study Group for Pediatric Liver Tumor 2 Protocol. JCO 2020, 38, 2488-2498. [CrossRef] [PubMed]

13. Kremer, N.; Walther, A.E.; Tiao, G.M. Management of hepatoblastoma: An update. Curr. Opin. Pediatrics 2014, $26,362-369$. [CrossRef]

14. Lim, I.I.P.; Bondoc, A.J.; Geller, J.I.; Tiao, G.M. Hepatoblastoma-The Evolution of Biology, Surgery, and Transplantation. Children 2018, 6, 1. [CrossRef] [PubMed]

15. Pham, T.A.; Gallo, A.M.; Concepcion, W.; Esquivel, C.O.; Bonham, C.A. Effect of Liver Transplant on Long-term Disease-Free Survival in Children with Hepatoblastoma and Hepatocellular Cancer. JAMA Surg. 2015, 150, 1150. [CrossRef]

16. Czauderna, P.; Otte, J.B.; Aronson, D.C.; Gauthier, F.; Mackinlay, G.; Roebuck, D.; Plaschkes, J.; Perilongo, G. Guidelines for surgical treatment of hepatoblastoma in the modern era-Recommendations from the Childhood Liver Tumour Strategy Group of the International Society of Paediatric Oncology (SIOPEL). Eur. J. Cancer 2005, 41, 1031-1036. [CrossRef]

17. Meyers, R.L. Liver transplantation in the management of unresectable hepatoblastoma in children. Front. Biosci. 2012, E4, 1293-1302. [CrossRef]

18. Ezekian, B.; Mulvihill, M.S.; Schroder, P.M.; Gilmore, B.F.; Leraas, H.J.; Gulack, B.C.; Commander, S.J.; Mavis, A.M.; Kreissman, S.G.; Stuart, J.; et al. Improved contemporary outcomes of liver transplantation for pediatric hepatoblastoma and hepatocellular carcinoma. Pediatr. Transplant. 2018, 22, e13305. [CrossRef]

19. Ismail, H.; Broniszczak, D.; Kaliciński, P.; Dembowska-Bagińska, B.; Perek, D.; Teisseyre, J.; Kluge, P.; Kościesza, A.; Lembas, A.; Markiewicz, M. Changing treatment and outcome of children with hepatoblastoma: Analysis of a single center experience over the last 20 years. J. Pediatric Surg. 2012, 47, 1331-1339. [CrossRef]

20. Angelico, R.; Grimaldi, C.; Gazia, C.; Saffioti, M.C.; Manzia, T.M.; Castellano, A.; Marco Spada, M. How Do Synchronous Lung Metastases Influence the Surgical Management of Children with Hepatoblastoma? An Update and Systematic Review of the Literature. Cancers 2019, 11, 1693. [CrossRef] [PubMed]

21. Shi, Y.; Commander, S.J.; Masand, P.M.; Heczey, A.; Goss, J.A.; Vasudevan, S.A. Vascular invasion is a prognostic indicator in hepatoblastoma. J. Pediatric Surg. 2017, 52, 956-961. [CrossRef] [PubMed]

22. Feng, J.; Polychronidis, G.; Heger, U.; Frongia, G.; Mehrabi, A.; Hoffmann, K. Incidence trends and survival prediction of hepatoblastoma in children: A population-based study. Cancer Commun. 2019, 39, 62. [CrossRef] [PubMed]

23. Kulkarni, S.; Brauer, D.G.; Turmelle, Y.; Stoll, J.; Nadler, M.; Chapman, W.C.; Doyle, M.B.; Khan, A.S. Surgical Therapy for Pediatric Hepatoblastoma in the USA over the Last Decade: Analysis of the National Cancer Database. J. Gastrointest. Cancer 2020, 52, 547-556. [CrossRef]

24. Otte, J.-B. Progress in the surgical treatment of malignant liver tumors in children. Cancer Treat. Rev. 2010, 36, 360-371. [CrossRef]

25. Meyers, R.L.; Czauderna, P.; Otte, J.-B. Surgical treatment of hepatoblastoma. Pediatr Blood Cancer 2012, 59, 800-808. [CrossRef]

26. Pimpalwar, A.P.; Sharif, K.; Ramani, P.; Stevens, M.; Grundy, R.; Morland, B.; Lloyd, C.; Kelly, D.A.; Buckles, J.A.; de Ville De Goyet, J. Strategy for hepatoblastoma management: Transplant versus nontransplant surgery. J. Pediatric Surg. 2002, 37, 240-245. [CrossRef]

27. Lautz, T.B.; Ben-Ami, T.; Tantemsapya, N.; Gosiengfiao, Y.; Superina, R.A. Successful nontransplant resection of POST-TEXT III and IV hepatoblastoma. Cancer 2011, 117, 1976-1983. [CrossRef] 
28. Meyers, R.L.; Tiao, G.M.; Dunn, S.P.; McGahren, E.D.; Langham, M.R. On behalf of the Central Surgical Review Committee, Children's Oncology Group AHEP-0731, Treatment of Children with All Stages Hepatoblastoma. Surgical management of children with locally advanced hepatoblastoma. Cancer 2012, 118, 4090-4091. [CrossRef] [PubMed]

29. Meyers, R.L.; Maibach, R.; Hiyama, E.; Häberle, B.; Krailo, M.; Rangaswami, A.; Aronson, D.C.; Malogolowkin, M.H.; Perilongo, G.; von Schweinitz, D.; et al. Risk-stratified staging in paediatric hepatoblastoma: A unified analysis from the Children's Hepatic tumors International Collaboration. Lancet Oncol. 2017, 18, 122-131. [CrossRef]

30. Otte, J.-B.; Meyers, R.L.; de Ville de Goyet, J. Transplantation for liver tumors in children: Time to (re)set the guidelines? Pediatr. Transplant. 2013, 17, 710-712. [CrossRef]

31. Otte, J.B.; Pritchard, J.; Aronson, D.C.; Brown, J.; Czauderna, P.; Maibach, R.; Perilongo, G.; Shafford, E.; Plaschkes, J. Liver transplantation for hepatoblastoma: Results from the International Society of Pediatric Oncology (SIOP) study SIOPEL-1 and review of the world experience. Pediatr. Blood Cancer 2004, 42, 74-83. [CrossRef] [PubMed]

32. Zsiros, J.; Brugieres, L.; Brock, P.; Roebuck, D.; Maibach, R.; Zimmermann, A.; Childs, M.; Pariente, D.; Laithier, V.; Otte, J.-B.; et al. Dose-dense cisplatin-based chemotherapy and surgery for children with high-risk hepatoblastoma (SIOPEL-4): A prospective, single-arm, feasibility study. Lancet Oncol. 2013, 14, 834-842. [CrossRef]

33. Zsíros, J.; Maibach, R.; Shafford, E.; Brugieres, L.; Brock, P.; Czauderna, P.; Roebuck, D.; Childs, M.; Zimmermann, A.; Laithier, V.; et al. Successful Treatment of Childhood High-Risk Hepatoblastoma with Dose-Intensive Multiagent Chemotherapy and Surgery: Final Results of the SIOPEL-3HR Study. JCO 2010, 28, 2584-2590. [CrossRef] [PubMed]

34. Özen, J.; Beime, J.; Brinkert, F.; Fischer, L.; Herden, U.; Grabhorn, E. Short- and long-term results of liver transplantation according to age at transplant: A single center experience of 351 children. Transplant. Int. 2021, 34, 1251-1260. [CrossRef]

35. Croome, K.P.; Wall, W.; Chandok, N.; Beck, G.; Marotta, P.; Hernandez-Alejandro, R. Inferior survival in liver transplant recipients with hepatocellular carcinoma receiving donation after cardiac death liver allografts. Liver Transplant. 2013, 19, $1214-1223$. [CrossRef] 